\title{
VARIATIONAL INTERPOLATION OF SUBSETS
}

\author{
JOHANNES WALLNER, HELMUT POTTMANN
}

\begin{abstract}
We consider the problem of variational interpolation of subsets of Euclidean spaces by curves such that the $L^{2}$ norm of the second derivative is minimized. It is well known that the resulting curves are cubic spline curves. We study geometric boundary conditions arising for various types of subsets such as subspaces, polyhedra, and submanifolds, and we indicate how solutions can be computed in the case of convex polyhedra.
\end{abstract}

\section{INTRODUCTION AND PREVIOUS WORK}

Cubic B-spline curves are frequently used for interpolation in Computer-Aided Geometric Design. One reason is that their definition and handling is rather simple, but they are flexible enough to allow interpolation with $C^{2}$ curves. The following is a well known property of the $C^{2}$ B-spline curves: If points $x_{0}, \ldots, x_{n} \in \mathbb{R}^{d}$ and real parameter values $u_{0}<\cdots<u_{n}$ are given, then there is a unique piecewise cubic $C^{2}$ curve $c(u)$ which assumes the values $c\left(u_{i}\right)=x_{i}$ (i.e., it interpolates the points $x_{i}$ ), and which has the additional property that the second derivatives $c^{\prime \prime}\left(u_{0}\right)$ and $c^{\prime \prime}\left(u_{n}\right)$ vanish. This cubic interpolant is at the same time the unique interpolant curve which minimizes the functional

$$
F(c)=\int\left\|c^{\prime \prime}(u)\right\|^{2} d u
$$

in some well-defined class of curves defined below (cf. [12]).

In the present paper we study a generalization of this problem: the variational interpolation of subsets $X_{0}, \ldots, X_{n}$ of Euclidean space $\mathbb{R}^{d}$. These subsets can be viewed as tolerance zones of points, and the variational interpolant of these subsets may be interpreted as interpolation of points within precisely defined error bounds. It turns out that the solution, to this problem, if it exists, is again a piecewise cubic curve.

An even more general problem is minimization of a functional of the above type with the same interpolation conditions, but with variable parameter values $u_{0}<\cdots<u_{N}$.

1991 Mathematics Subject Classification. 41A29, 65D17, 53A04.

Key words and phrases. Variational interpolation, cubic splines. 
Previous work on these topics includes $[3,4,5,6]$, where a functional of the form

$$
\int\left\|c^{(m)}(t)\right\|^{2} d t
$$

is minimized, where $c$ ranges in the Sobolev space $L_{2}^{(m)}\left([0,1], \mathbb{R}^{d}\right)$ of $\mathbb{R}^{d}$ valued curves wich satisfy the interpolation conditions $c^{(j)}\left(u_{i}\right) \in K_{i j}$, for certain subsets $K_{i j}$. The more general problem mentioned above is also discussed in these papers. The main results concern existence of a solution, and 'optimality conditions' which allows one to recognize solutions among the set of piecewise cubic interpolants. The solution curves are interpreted as smoothing splines, and the optimality conditions involve the smoothing spline weights.

The present paper deals with two topics: One is geometrically characterizing of interpolants of affine subspaces and of smooth submanifolds $X_{i}$, possibly with boundary, which make the functional $F$ of Equ. (1) stationary. The other is description and computation of the set of minimizing interpolants, if the subsets $X_{i}$ to be interpolated are affine subspaces or polyhedra.

\section{Definitions}

We write $f \in A C([a, b])$ for an absolutely continuous function $f$ : $[a, b] \rightarrow \mathbb{R}^{d}$. As usual we identify functions which are equal almost everywhere, so that we can write, for instance $f \in L^{2}$. Recall that $f \in A C([a, b])$ implies that $f$ is differentiable almost everywhere, and that $f(x)-f(a)=\int_{a}^{x} f^{\prime}(t) d t$ (see e.g. [11]).

We assume that we are given real parameter values $u_{0}<\cdots<u_{n}$, and let $I=\left[u_{0}, u_{n}\right]$. We consider the linear space $\mathcal{C}$ of curves defined by

$$
c \in \mathcal{C} \Longleftrightarrow c: I \rightarrow \mathbb{R}^{d}, c^{\prime} \in A C(I), c^{\prime \prime} \in L^{2}(I) .
$$

If $X_{0}, \ldots, X_{n}$ are subsets of $\mathbb{R}^{d}$, then we are looking for those curves $c \in \mathcal{C}$ which assume values $c\left(u_{i}\right) \in X_{i}$ for $i=0, \ldots, n$, and among them, for those which minimize the functional

$$
F(c)=\int_{u_{0}}^{u_{n}}\left\|c^{\prime \prime}\right\|^{2} .
$$

We refer to them as minimizing interpolants of the subsets $X_{0}, \ldots, X_{n}$. Further, we look for curves which make the functional (4) stationary (if the subsets in question are smooth) without necessarily minimizing it.

The property of cubic $C^{2}$ B-splines mentioned at the beginning could be expressed now by saying that the minimizing interpolant of points $x_{0}, \ldots, x_{n}$ is the piecewise cubic $C^{2}$ curve with $c^{\prime \prime}\left(u_{0}\right)=c^{\prime \prime}\left(u_{n}\right)=0$ and $c\left(u_{i}\right)=x_{i}$. 


\section{VARiational Interpolation of Subsets}

3.1. Affine subspaces of $\mathbb{R}^{d}$. We first study the case that the subsets $X_{0}, \ldots, X_{n}$ are affine subspaces, such as points, lines, and planes. We use the notation

$$
\Delta_{u_{0}} f=\lim _{u \searrow u_{0}} f-\lim _{u \nearrow u_{0}} f
$$

for the jump of a piecewise continuous function at $u=u_{0}$.

Lemma 1. Assume that $X_{0}, \ldots, X_{n}$ are affine subspaces of $\mathbb{R}^{d}$, and assume further that a curve $c \in \mathcal{C}$ has the property $c\left(u_{i}\right) \in X_{i}$ for $i=0, \ldots, n$. If $c$ satisfies the following three conditions:

(a) The restrictions $c \mid\left[u_{i}, u_{i+1}\right]$ are polynomial of degree $\leq 3$;

(b) $c$ is $C^{2}$ with $c^{\prime \prime}\left(u_{0}\right)=c^{\prime \prime}\left(u_{n}\right)=0$;

(c) $\Delta_{u_{i}} c^{\prime \prime \prime}$ is orthogonal to $X_{i}$ for $i=1, \ldots, n-1$. The vectors $c^{\prime \prime \prime}\left(u_{0}\right), c^{\prime \prime \prime}\left(u_{n}\right)$ are orthogonal to $X_{0}, X_{n}$, respectively;

then $c$ minimizes the functional $F$ in $\mathcal{C}$, and all interpolating minimizers are of this form.

Proof. We endow $\mathcal{C}$ with the inner product

$$
\langle c, \bar{c}\rangle=\int_{u_{0}}^{u_{n}}\left\langle c^{\prime \prime}(u), \bar{c}^{\prime \prime}(u)\right\rangle d u, \quad \text { so } \quad F(c)=\langle c, c\rangle .
$$

The scalar product $\left\langle c^{\prime \prime}, \bar{c}^{\prime \prime}\right\rangle$ is to be taken in the Euclidean vector space $\mathbb{R}^{d}$. We have

$$
\langle\bar{c}, \bar{c}\rangle-\langle c, c\rangle=2\langle c, \bar{c}-c\rangle+\langle\bar{c}-c, \bar{c}-c\rangle
$$

for all $c, \bar{c} \in \mathcal{C} .\langle$,$\rangle is positive semi-definite, so \langle c, \bar{c}-c\rangle=0$ implies that $F(\bar{c}) \geq F(c)$, and $\langle c, \bar{c}-c\rangle>0$ implies that $F(\bar{c})>F(c)$.

We assume that $\bar{c}$ interpolates $X_{0}, \ldots, X_{n}$ in the same way as $c$ does. We let $V(u)=\bar{c}(u)-c(u)$ and compute $\langle c, \bar{c}-c\rangle$ by integration by parts in the intervals $\left[u_{0}, u_{1}\right],\left[u_{1}, u_{2}\right], \ldots$ and summing up:

$$
\begin{aligned}
\langle c, \bar{c}-c\rangle & =\int\left\langle c^{\prime \prime}(u), V^{\prime \prime}(u)\right\rangle d u \\
& \left.=\int\left\langle c^{\prime \prime \prime \prime}, V\right\rangle d u+\left\langle c^{\prime \prime}\left(u_{n}\right), V^{\prime}\left(u_{n}\right)\right\rangle-\left\langle c^{\prime \prime}\left(u_{0}\right), V^{\prime}\left(u_{0}\right)\right)\right\rangle \\
& +\left\langle c^{\prime \prime \prime}\left(u_{0}\right), V\left(u_{0}\right)\right\rangle-\left\langle c^{\prime \prime \prime}\left(u_{n}\right), V\left(u_{n}\right)\right\rangle+\sum_{i=2}^{n-1}\left\langle\Delta_{u_{i}} c^{\prime \prime \prime}, V\left(u_{i}\right)\right\rangle .
\end{aligned}
$$

As $V\left(u_{i}\right)$ is parallel to $X_{i}$, conditions (a)-(c) immediately imply that $\langle c, \bar{c}-c\rangle=0$, which shows the sufficiency of (a)-(c).

Any other minimizer $\bar{c}$ must satisfy $\langle c-\bar{c}, c-\bar{c}\rangle=0$, because otherwise we would have $F(\bar{c})>F(c)$. This condition implies that $\int\left\|(\bar{c}-c)^{\prime \prime}\right\|=0$, that is, $(c-\bar{c})^{\prime \prime}=0$ almost everywhere. As $(c-\bar{c})^{\prime} \in$ $A C\left(\left[u_{0}, u_{n}\right]\right)$, we have $(c-\bar{c})^{\prime}(u)-(c-\bar{c})^{\prime}\left(u_{0}\right)=\int_{u_{0}}^{u}(c-\bar{c})^{\prime \prime}=0$. It 
follows that $c-\bar{c}$ is an inhomogeneous linear function. Consequently, $c^{\prime \prime}=\bar{c}^{\prime \prime}$, and $\bar{c}$ satisfies (a)-(c) if and only if $c$ does.

We consider the subspace $\mathcal{P}^{3}$ of $\mathcal{C}$ which is defined by

$$
\begin{aligned}
c \in \mathcal{P}^{3} \Longleftrightarrow & c \in C^{2}\left(\left[u_{0}, u_{n}\right]\right), \text { and } c \mid\left[u_{i}, u_{i+1}\right] \text { is } \\
& \text { polynomial of degree } \leq 3 \text { for } i=0, \ldots, n-1 .
\end{aligned}
$$

Recall that a cubic curve $c$ is uniquely determined by its values and derivatives at two different parameter values. If $c \in \mathcal{P}^{3}$, then $c, c^{\prime}$, $c^{\prime \prime}$ are continuous, so every cubic segment leaves exactly $d$ degrees of freedom for its successor. Therefore,

$$
\operatorname{dim} \mathcal{P}^{3}=4 d+(n-1) d=(n+3) d .
$$

We now derive a partial result concerning the existence and uniqueness of the variational interpolant.

Lemma 2. We use the notation of Lemma 1. Assume that $X_{i}=p_{i}+U_{i}$ are affine subspaces of $\mathbb{R}^{d}$ parallel to linear subspaces $U_{i}$. It might happen that there exist $a_{0}, a_{1} \in \mathbb{R}^{d}$, not both zero, such that

$$
a_{0}+u_{i} a_{1} \in U_{i}, \quad(i=0, \ldots, n) .
$$

If this is not the case, then the variational interpolation problem is uniquely solvable within the class $\mathcal{C}$, and satisfies (a)-(c) of Lemma 1. Especially, the minimizing interpolant is in $\mathcal{P}^{3}$.

Proof. We show that conditions (a)-(c) of Lemma 1 are satisfied by a unique curve in $\mathcal{P}^{3}$. We use the fact that a system of $\left(\operatorname{dim} \mathcal{P}^{3}\right)$ linear equations is uniquely solvable if and only if 0 is the only solution of the corresponding homogeneous system.

The interpolation conditions $c\left(u_{i}\right) \in X_{i}$, the orthogonality conditions (b), and the end conditions (c) represent

$$
\sum_{i=0}^{n}\left(d-\operatorname{dim} X_{i}\right), \quad \sum_{i=0}^{n} \operatorname{dim} X_{i}, \quad 2 d
$$

inhomogeneous linear equations for the curve $c \in \mathcal{P}^{3}$, respectively. The total number of equations is $(n+3) d=\operatorname{dim} \mathcal{P}^{3}$. If we replace the spaces $X_{i}$ by $U_{i}$, we get the corresponding homogeneous system of linear equations.

We have to show that $c(u)=0$ is the only minimizing interpolant of the subspaces $U_{0}, \ldots, U_{n}$. A possible minimizing interpolant $c$ must satisfy $F(c)=F(0)=0$, which implies that $c$ is piecewise linear (the argumentation is the same as in the proof of Lemma 1).

As $c$ is $C^{2}$, it is actually of the form $c(u)=a_{0}+u a_{1}$, with $a_{0}, a_{1} \in$ $\mathbb{R}^{d}$. This means that $a_{0}+u_{i} a_{1} \in U_{i}$, which was forbidden by our assumptions. 


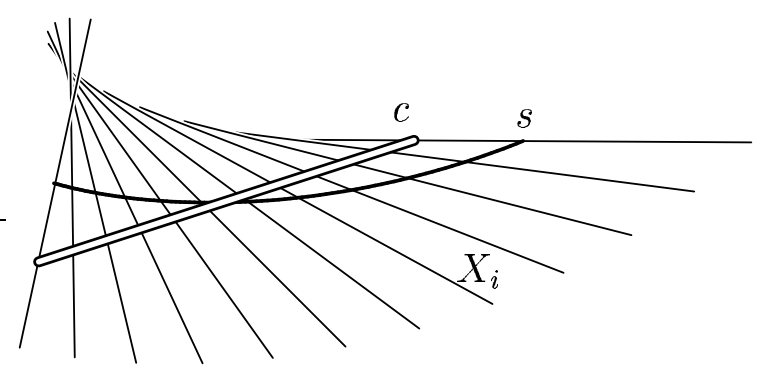

Figure 1. Minimizing interpolant of the lines $X_{i}$.

Example 1. Fig. 1 (cf. [10], p. 301) shows the unique variational interpolant of lines $X_{i}$. These lines are generator lines $u=u_{i}$ of a ruled surface of the form

$$
s(u, v)=l(u)+v r(u) .
$$

In this example $l$ is a linearly parametrized line. The variational interpolation must, of course, reproduce this line. The curve $s$ also shown in Fig. 1 is the striction curve. This is a Euclidean differential invariant of ruled surfaces. A definition which does not need any further notions of differential geometry is that the striction curve is the locus of points where the generator lines $u=c=$ const. are closest to their 'neighbours' $u=c+\Delta u$ as $\Delta u \rightarrow 0$ (see [10], pp. 264 and 285).

An explicit system of linear equations describing the variational interpolation of lines in Euclidean three-space is given in [10], p. 300f. Sec. 5.4.3 of [10] discusses the variational interpolation of ruled surfaces, and shows a possible solution which contains variational interpolation of lines by a curve as a subproblem.

3.2. Reduction to the cubic case. Lemma 2 includes the following well known elementary result as a special case: The variational interpolation problem within $\mathcal{C}$ for points $p_{0}=X_{0}, \ldots, p_{n}=X_{n}$ is uniquely solvable. There is exactly one curve $c \in \mathcal{P}^{3}$ with $c\left(u_{i}\right)=p_{i}$ and $c^{\prime \prime}\left(u_{0}\right)=c^{\prime \prime}\left(u_{n}\right)=0$. This curve will be denoted by

$$
I\left(p_{0}, \ldots, p_{n}\right) .
$$

A very similar (and well known) result is the following: For all points $p_{0}, \ldots, p_{n} \in \mathbb{R}^{d}$, and vectors $w_{0}, w_{n} \in \mathbb{R}^{d}$, there exists a unique $C^{2}$ piecewise cubic curve $c \in \mathcal{P}^{3}$ with $c\left(u_{i}\right)=p_{i}(i=0, \ldots, n)$ and $c^{\prime}\left(u_{0}\right)=$ $w_{0}, c^{\prime}\left(u_{n}\right)=w_{n}$. It will be denoted by

$$
\widetilde{I}\left(p_{0}, \ldots, p_{n} ; w_{0}, w_{n}\right) .
$$

Both $I$ and $\widetilde{I}$ are found by solving a linear system of equations whose matrix depends on the knot values $u_{i}$, but not on the points $p_{i}$ or the vectors $w_{0}, w_{n}$. Thus we have 
Lemma 3. The mappings $I:\left(\mathbb{R}^{d}\right)^{n+1} \rightarrow \mathcal{P}^{3}$ and $\widetilde{I}:\left(\mathbb{R}^{d}\right)^{n+3} \rightarrow \mathcal{P}^{3}$ are linear.

We reduce the problem of interpolating within the class $\mathcal{C}$ to the problem of interpolating within the class $\mathcal{P}^{3}$. Obviously, if $X_{1}, \ldots, X_{n}$ are subsets of $\mathbb{R}^{d}$, and $c$ is a curve in $\mathcal{C}$ with $c\left(u_{i}\right) \in X_{i}$, then

$$
\begin{aligned}
& F(c) \geq F\left(I\left(c\left(u_{0}\right), \ldots, c\left(u_{n}\right)\right)\right), \quad \text { and } \\
& \inf \left\{F(c) \mid c \in \mathcal{C}, c\left(u_{i}\right) \in X_{i}\right\}=\inf \left\{F(c) \mid c \in \mathcal{P}^{3}, c\left(u_{i}\right) \in X_{i}\right\}
\end{aligned}
$$

We consider the set $V=\left(\mathbb{R}^{d}\right)^{n+1}$ (which is the $(n+1)$ )-fold product of the affine space $\mathbb{R}^{d}$ with itself) and define a quadratic form in $V$ by letting

$$
q\left(p_{0}, \ldots, p_{n}\right)=F\left(I\left(p_{0}, \ldots, p_{n}\right)\right)
$$

By (16), finding a minimizing interpolant of subsets $X_{0}, \ldots, X_{n}$ is equivalent to minimizing the function $q$ on $X_{0} \times \cdots \times X_{n}$.

Remark: From the structural viewpoint, $\mathbb{R}^{d}$ is here considered as an affine space, and $\left(\mathbb{R}^{d}\right)^{n+1}$ is a product of $n+1$ copies of this affine space. A translation $x \mapsto x+a$ of $\mathbb{R}^{d}$ gives rise to a transformation $\left(x_{0}, \ldots, x_{n}\right) \mapsto\left(x_{0}+a, \ldots, x_{n}+a\right)$ of $V$. The quadratic form $q$ is invariant with respect to the group of these transformations, because $F$ is invariant with respect to translations.

3.3. Submanifolds of Euclidean space. We are going to investigate interpolants of curves and surfaces, i.e., submanifolds, which make the functional $F$ stationary without necessarily minimizing it. The answer will be an infinitesimal condition involving the first three derivatives of the curve. A characterization of an actual (global) minimum is not possible with conditions of that type: it must use the shape of the sets $X_{i}$ (e.g., being convex or being a subspace). An infinitesimal characterization of local minima is possible and uses higher derivatives. Thus, for the sake of generality, we consider the local situation only, and for the sake of simplicity, we are content with a first order analysis.

More precisely, we call a bivariate smooth function $c_{v}(u)$ an admissible variation of $c$ if it is defined for $v$ in a neighbourhood of 0 and $u \in\left[u_{0}, u_{N}\right]$, if $c_{0}=c$ and the interpolation condition $c_{v}\left(u_{i}\right) \in X_{i}$ is satisfied for all $v$ and $i=0, \ldots, n$. Then $c$ makes $F$ stationary if for all admissible variations the derivative

$$
\left.\frac{d}{d v} F\left(c_{v}\right)\right|_{v=0}=0
$$

Theorem 4. Assume that $X_{0}, \ldots, X_{n}$ are smooth submanifolds of $\mathbb{R}^{d}$. $A$ curve in $\mathcal{P}^{3}$ makes the functional $F$ stationary within the set of $\mathcal{P}^{3}$ interpolants of $X_{0}, \ldots, X_{n}$ if and only if the following conditions are satisfied: 
(a) $\Delta_{u_{i}} c^{\prime \prime \prime}$ is orthogonal to the tangent space $T_{c\left(u_{i}\right)} X_{i}$ for $i=1, \ldots$, $n-1$, and $c^{\prime \prime \prime}\left(u_{0}\right), c^{\prime \prime \prime}\left(u_{n}\right)$ are orthogonal to $T_{c\left(u_{0}\right)} X_{i}, T_{c\left(u_{n}\right)} X_{i}$, respectively.

(b) $c^{\prime \prime}\left(u_{0}\right)=c^{\prime \prime}\left(u_{n}\right)=0$, i.e., $c=I\left(c\left(u_{0}\right), \ldots, c(n)\right)$.

Proof. Consider an admissible variation $c_{v}(u)$ of $c$ within $\mathcal{P}^{3}$, and let $V(u)=\partial c_{v}(u) /\left.\partial v\right|_{v=0}$. We use integration by parts to compute

$$
\begin{aligned}
\left.\frac{1}{2} \frac{d F\left(c_{v}\right)}{d v}\right|_{v=0} & =\left\langle V^{\prime}\left(u_{n}\right), c^{\prime \prime}\left(u_{n}\right)\right\rangle-\left\langle V^{\prime}\left(u_{0}\right), c^{\prime \prime}\left(u_{0}\right)\right\rangle-\left\langle V\left(u_{n}\right), c^{\prime \prime \prime}\left(u_{n}\right)\right\rangle \\
& +\left\langle V\left(u_{0}\right), c^{\prime \prime \prime}\left(u_{0}\right)\right\rangle+\sum_{i=1}^{n-1}\left\langle\Delta_{u_{i}} c^{\prime \prime \prime}, V\left(u_{i}\right)\right\rangle .
\end{aligned}
$$

Obviously, if (a) and (b) are satisfied, $F$ is stationary at $c=c_{0}$. To show the converse, we argue as follows:

For a given $c=c_{0}$, we try to find a variation $c_{v}(u)$ with $V\left(u_{i}\right)=0$, but $V^{\prime}\left(u_{0}\right)=c^{\prime \prime}\left(u_{0}\right)$ and $V^{\prime}\left(u_{n}\right)=c^{\prime \prime}\left(u_{n}\right)$. Then the derivative of $F\left(c_{v}\right)$ will be positive unless $c^{\prime \prime}\left(u_{0}\right)=c^{\prime \prime}\left(u_{n}\right)$. To find $c_{v}$, we choose curves $w_{0}(v)$ and $w_{n}(v)$ in $\mathbb{R}^{d}$, such that $d w_{0} / d v(0)=c^{\prime \prime}\left(u_{0}\right)$, and $d w_{n} / d v(0)=c^{\prime \prime}\left(u_{n}\right)$. We let

$$
c_{v}=\widetilde{I}\left(c\left(u_{0}\right), \ldots, c\left(u_{n}\right) ; w_{0}(v), w_{n}(v)\right) .
$$

By construction, $c_{v}$ has the desired properties, and its existence shows that necessarily $c^{\prime \prime}\left(u_{0}\right)=c^{\prime \prime}\left(u_{n}\right)=0$, if $c$ is to make $F$ stationary.

To show (a), we choose curves $p_{i}(v)$ in $X_{i}(i=0, \ldots, n)$, such that $p_{0}(0)=c\left(u_{0}\right), \ldots, p_{n}(0)=c\left(u_{n}\right)$, and such that the tangent vectors $d p_{0} / d v(0), \ldots, d p_{n} / d v(0)$ equal the orthogonal projections of the vectors $c^{\prime \prime \prime}\left(u_{0}\right), \Delta_{u_{1}} c^{\prime \prime \prime}, \ldots, \Delta_{u_{n-1}} c^{\prime \prime \prime}, c^{\prime \prime \prime}\left(u_{n}\right)$ onto the respective tangent spaces $T_{c\left(u_{i}\right)} X_{i}$. Finally, we let

$$
c_{v}(u)=I\left(p_{0}(v), \ldots, p_{n}(v)\right) .
$$

By $(\mathrm{b}), c_{0}=c$, so $c_{v}$ is actually an admissible variation. By construction, $d F\left(c_{v}\right) / d v$ is nonzero unless (a) is satisfied. This concludes the proof.

This result on the jump of the third derivative yields a geometric interpretation of the conditions given in the section 'optimality conditions for fixed data sites' of [4] and the condition in [6]. These two papers give conditions on the weights of the solution curves when interpreted as smoothing splines.

Theorem 5. An interpolant $c$ of $X_{0}, \ldots, X_{n}$ makes $F$ stationary in the class $\mathcal{C}$ if and only if $c \in \mathcal{P}^{3}$ and $c$ makes $F$ stationary within $\mathcal{P}^{3}$.

Proof. If $c \notin \mathcal{P}^{3}$, then we consider the curve $\bar{c}=I\left(c\left(u_{0}\right), \ldots, c\left(u_{n}\right)\right)$. Clearly, $F(c)>F(\bar{c})$. Consider the admissible variation $c_{v}(u)=$ $(1-v) c(u)+v \bar{c}(u)$. The mapping $v \mapsto F\left(c_{v}\right)$ is the restriction of the positive semidefinite quadratic form $F$ to a straight line (and therefore 
a quadratic function). It does not assume its minimum at $v=0$, so we have $d F\left(c_{v}\right) / d v \neq 0$ for $v=0$. This implies the 'if' part of the statement.

The 'only if' part is shown as follows: Assume that $c \in \mathcal{P}^{3}$, and that $c$ makes $F$ stationary within $\mathcal{P}^{3}$. For an admissible variation $c_{v}$ of $c$ within $\mathcal{C}$, consider $\bar{c}_{v}=I\left(c_{v}\left(u_{0}\right), \ldots, c_{v}\left(u_{n}\right)\right)$. By Th. $4, c_{0}=\bar{c}_{0}$, so $\bar{c}_{v}$ is an admissible variation of $c$ within $\mathcal{P}^{3}$. Now

$$
\frac{d F\left(\bar{c}_{v}\right)}{d v}(0)=0, F\left(c_{v}\right) \geq F\left(\bar{c}_{v}\right) \Longrightarrow \frac{d F\left(c_{v}\right)}{d v}(0)=0 .
$$

3.4. Submanifolds with boundary. We now consider the case that $X_{0}, \ldots, X_{n}$ are submanifolds with boundary. These are defined as subsets of $\mathbb{R}^{d}$, which are locally diffeomorphic to polyhedra contained in real vector spaces of varying dimensions. The $r$-dimensional facets of those polyhedral correspond, via these local diffeomorphisms, to $r$-dimensional boundary components of $X_{i}$. Such boundary components are submanifolds with boundary themselves. The points in the interior of the above mentioned polyhedra correspond, by definition, to interior points of $X_{i}$ (the set of interior points being a smooth submanifold without boundary). By construction, all points of $X_{i}$ are interior points of some boundary component, if for the sake of consistency we define that 0 -dimensional submanifolds contain only interior points. In order to make the tangent space of a point unique, we require that the tangent space $T_{p} X_{i}$ of a point $p \in X_{i}$ is the tangent space (in the ordinary sense) of that $r$-dimensional boundary component the point $p$ is an interior point of.

Theorem 6. Assume that $X_{1}, \ldots, X_{n}$ are submanifolds with boundary, and that the remaining assumptions are as in Th. 4. The results of both Th. 4 and Th. 5 remain valid if we adopt the definition of tangent space given above.

Proof. If $c$ is an interpolant of $X_{0}, \ldots, X_{n}$, and $c_{v}(u)$ its admissible variation, consider the vector field $V(u)$ as defined in the proof of Th. 4. If $c\left(u_{i}\right)$ is a boundary point and $V\left(u_{i}\right) \notin T_{c\left(u_{i}\right)} X_{i}$, then the curve $v \mapsto c_{v}\left(u_{i}\right)$ leaves $X_{i}$ in all neighbourhoods of 0 . Admissible variations therefore have the property that

$$
V\left(u_{i}\right) \in T_{c\left(u_{i}\right)} X_{i} .
$$

In order to show Th. 4 , we note that we can replace $X_{i}$ by that boundary component $\widetilde{X}_{i}$ which contains $c\left(u_{i}\right)$ as an interior point: The derivative of $F\left(c_{v}\right)$ depends on the vectors $V\left(u_{i}\right)$ only, and for all admissible variations $c_{v}(u)$ there is an admissible variation which has the same vectors $V\left(u_{i}\right)$, and where the curve $c_{v}\left(u_{i}\right)$ is entirely contained in $\widetilde{X}_{i}$. This shows that Th. 4 remains valid. The proof of Th. 5 does not need any changes. 


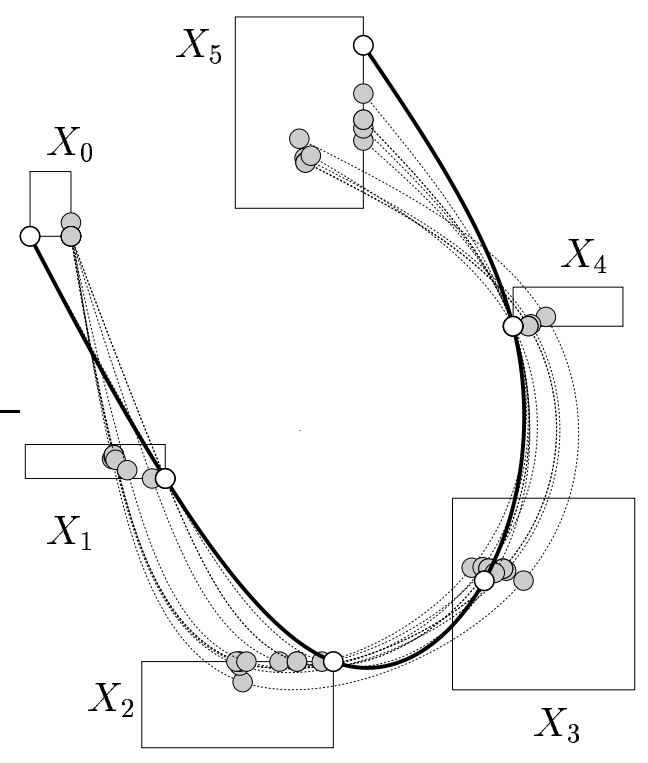

FiguRE 2. Variational interpolation of rectangles $X_{i}$ in the Euclidean plane.

Example 2. We use Fig. 2 to describe the effects of Th. 6: The bold curve $c(u)$ variationally interpolates the rectangles $X_{0}, \ldots, X_{n}$. If $c\left(u_{i}\right)$ is contained in the interior of the rectangle $X_{i}$, Th. 6 says that there is no jump in $c^{\prime \prime \prime}\left(u_{i}\right)$ at all (only the zero vector is orthogonal to the entire plane). This implies that the two cubic segments which meet at $c\left(u_{i}\right)$ are parts of the same cubic curve.

If $c\left(u_{i}\right)$ is contained in an edge, the jump in $c^{\prime \prime \prime}$ is orthogonal to that edge. If $c\left(u_{i}\right)$ is a vertex of $X_{i}$, then no restriction is imposed on $\Delta c^{\prime \prime \prime}\left(u_{i}\right)$ by Th. 6 (all vectors are orthogonal to the zero subspace $\left.T_{c\left(u_{i}\right)} X\right)$.

\section{Existence AND UNIQUENESS OF THE MINIMIZING INTERPOLANT}

4.1. Generalities. Existence of solutions to the more general variational interpolation problem for subsets with non-fixed knot values $u_{i}$ has been discussed in [3] and [5]. It is shown there that a solution exists of the subsets to be interpolated are closed and a certain number of them are bounded. Here we consider the existence and uniqueness problem from a slightly different (and more elementary) point of view, and with special attention to subspaces and polyhedra (which do not have to be bounded).

We have already seen that minimizing $F$ within $\mathcal{C}$ amounts to minimizing $q$ within the product space $V=\left(\mathbb{R}^{d}\right)^{n+1}$ (which implies immediately that the minimization problem is solvable whenever the sets $X_{i}$ are compact). The scalar product $\langle$,$\rangle in the product space$ $V=\left(\mathbb{R}^{d}\right)^{n+1}$ defined by the quadratic form $q$ is positive semidefinite. 
Recall that in this case the radical

$$
V^{\perp}=\{v \in V \mid\langle v, x\rangle=0 \forall x \in V\},
$$

coincides with the set of isotropic vectors

$$
q^{-1}(0)=\{v \in V \mid\langle v, v\rangle=0\} .
$$

Lemma 7. The radical $V^{\perp}$ consists of vectors $\left(p_{0}, \ldots, p_{n}\right)$ of the form $p_{i}=a_{0}+u_{i} a_{1}$, where $a_{0}, a_{1} \in \mathbb{R}^{d} . \operatorname{dim} V^{\perp}=2 d$.

Proof. $q(p)=0$ means that the cubic minimizing $C^{2}$ interpolant of the points $p_{0}, \ldots, p_{n}$ is linearly parametrized.

The scalar product $\langle$,$\rangle becomes well-defined and positive definite$ in the factor space $V / V^{\perp}$, if we define $\left\langle a+V^{\perp}, b+V^{\perp}\right\rangle=\langle a, b\rangle$. Then the projection $v \mapsto v+V^{\perp}$ of $V$ onto the factor space $V / V^{\perp}$ does not change scalar products. Likewise projections parallel to $V^{\perp}$ onto any complementary subspace (all of them are isometric to $V / V^{\perp}$ ) do not change scalar products. Fix any of these projections and call it $\pi$. We have

$$
\operatorname{ker} \pi=V^{\perp}, \operatorname{dim} \pi(V)=d(n-1) .
$$

The scalar product and quadratic form in $\pi(V)$ which are defined by $\langle$,$\rangle and q$ are denoted by the same letters. By construction, $q$ is positive definite in $\pi(V)$.

Lemma 8. Consider subsets $X_{0}, \ldots, X_{n}$ of $\mathbb{R}^{d}$. If $\pi\left(X_{0} \times \cdots \times X_{n}\right)$ is closed, then the variational interpolation problem with input data $X_{0}, \ldots, X_{n}$ has a solution $c$. If $F(c)=\rho$, then the solution curves have the form $I\left(p_{0}, \ldots, p_{n}\right)$, where $\left(p_{0}, \ldots, p_{n}\right)$ ranges in the set $q^{-1}(\rho) \cap$ $\left(X_{0} \times \cdots \times X_{n}\right)$.

Proof. The closed $q$-unit balls in $\pi(V)$ are compact, so $q$ has a minimum $\rho$ on any closed set.

\subsection{Examples.}

Theorem 9. If $X_{0}, \ldots, X_{n}$ are affine subspaces, the variational interpolation problem is solvable. Assume that $X_{i}=p_{i}+U_{i}$, with linear subspaces $U_{i}$. Let

$$
\begin{aligned}
W & =V^{\perp} \cap\left(U_{0} \times \cdots \times U_{n}\right) \\
& =\left\{\left(x_{0}, \ldots, x_{n}\right) \mid \exists a_{0}, a_{1} \in \mathbb{R}^{d}: x_{i} \in U_{i}, x_{i}=a_{0}+a_{1} u_{i}\right\} .
\end{aligned}
$$

Then the minimizing variational interpolants of $X_{0}, \ldots, X_{n}$ have the form $I\left(x_{0}, \ldots, x_{n}\right)$, where $\left(x_{0}, \ldots, x_{n}\right)$ ranges in an affine subspace parallel to $W$.

Proof. $\pi\left(X_{0} \times \cdots \times X_{n}\right)$ is an affine subspace and therefore closed. By Lemma 8, the problem is solvable. As the proof of Lemma 2 shows, the solution depends on solving a system of linear equations. We already know that this system is solvable. The solution of the corresponding 
homogeneous system is described in the proof of Lemma 2. By Lemma 7, which explicitly describes $V^{\perp}$, this solution is given by $W$.

Example 3. Th. 9 is illustrated in Fig. 3 (cf. [10], p. 301). Straight lines $X_{i}=p_{i}+U_{i}$ are arranged such that the one-dimensional subspaces $U_{i}$ are spanned by vectors $a_{0}+u_{i} a_{1}$, with some $a_{0}, a_{1} \in \mathbb{R}^{3}$. Here $a_{0}, a_{1}$ are unique up to a common scalar multiple. This implies that the intersection $V^{\perp} \cap\left(U_{0} \times \cdots \times U_{n}\right)$ mentioned in the theorem has dimension one, and there is a one-parameter linear family of minimizing interpolants.

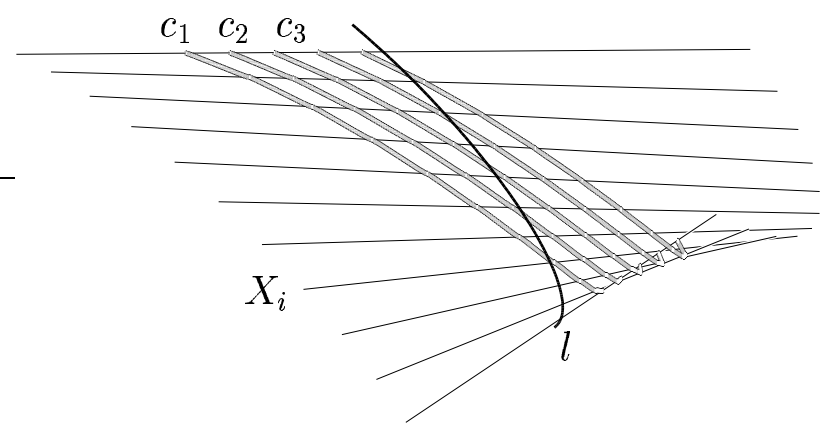

Figure 3. A one-parameter family $c_{i}$ of minimizing interpolants of lines $X_{i}$ The lines are taken from a smooth ruled surface which contains the curve $l$.

Corollary 10. If $X_{0}, \ldots, X_{n}$ are polyhedra (with finitely many faces, possibly unbounded), the variational interpolation problem is solvable.

Proof. $\pi\left(X_{0} \times \cdots \times X_{n}\right)$ is a polyhedron and therefore closed.

4.3. Convex polyhedra and intervals. Convex polyhedra are defined as the convex hulls of a finite number of points and closed rays. They are closed subsets of $\mathbb{R}^{d}$, and their products and projections are again convex polyhedra.

Theorem 11. If $X_{0}, \ldots, X_{n}$ are convex polyhedra, the variational interpolation problem has a solution $c=I\left(p_{0}, \ldots, p_{n}\right)$ with $p_{i} \in X_{i}$. All solutions are of the form $I\left(x_{0}, \ldots, x_{n}\right)$, where

$$
\left(x_{0}, \ldots, x_{n}\right) \in\left(\left(p_{0}, \ldots, p_{n}\right)+V^{\perp}\right) \cap\left(X_{0} \times \cdots \times X_{n}\right) .
$$

Proof. The existence of $c$ has already been stated by Cor. 10. To describe the set of solutions, we use Lemma 8. Let $\rho=F(c)$. If $\rho=0$, all solutions are of the form $I\left(x_{0}, \ldots, x_{n}\right)$ with $q\left(x_{0}, \ldots, x_{n}\right)=0$. As $q^{-1}(0)=V^{\perp}$, Equ. (29) follows.

If $\rho \neq 0, q^{-1}(\rho)$ is a cylinder with generator subspaces parallel to $V^{\perp}$. The set $q^{-1}([0, \rho])$ is convex and $q^{-1}(\rho)$ is its boundary. As the set $q^{-1}([0, \rho])+V^{\perp}$ is a strictly convex subset of $V / V^{\perp}$, other solutions $I\left(x_{0}, \ldots, x_{n}\right)$ can differ from $I\left(p_{0}, \ldots, p_{n}\right)$ only in the way that $\left(p_{0}-\right.$ $\left.x_{0}, \ldots, p_{n}-x_{n}\right) \in V^{\perp}$. 


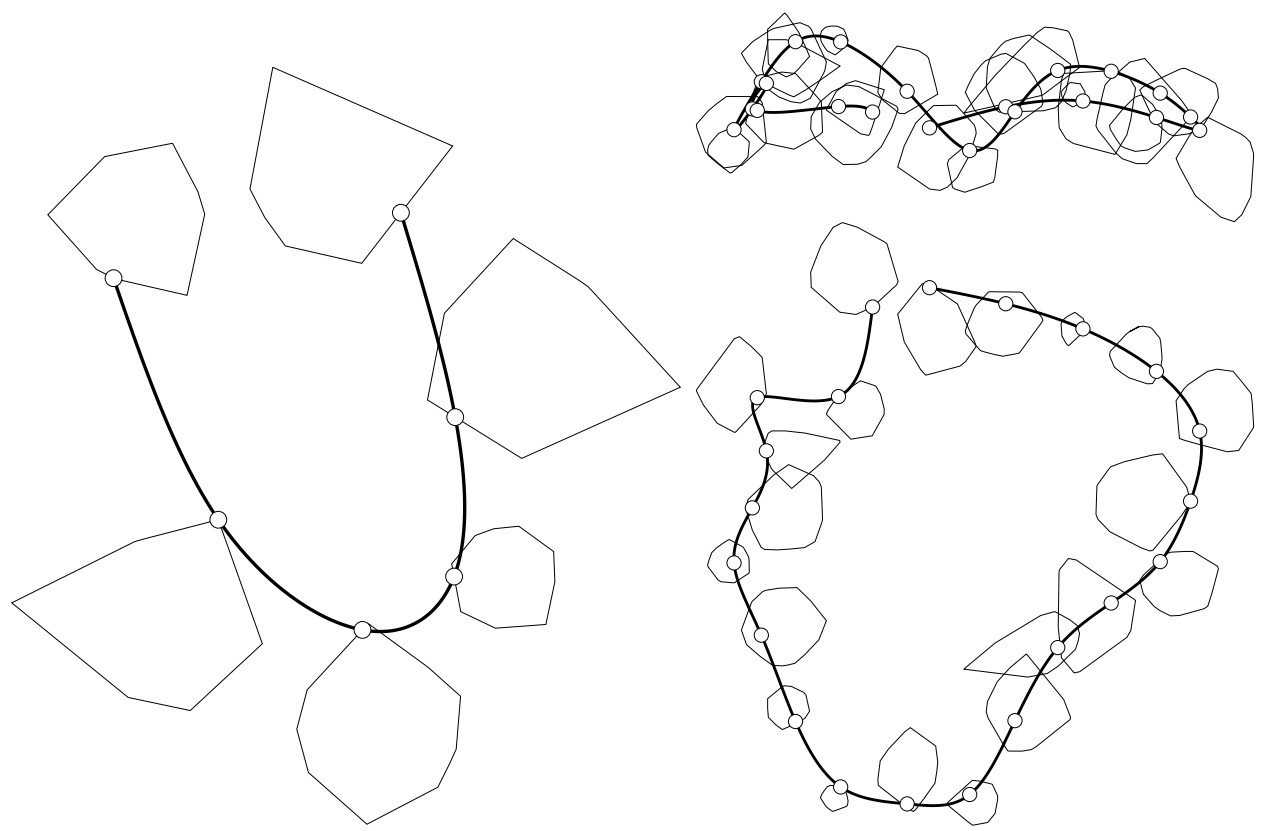

FigURE 4. Interpolation of convex polygons in the plane (left) and convex polyhedra in three-space. Top view (right, bottom) and front view (right, top).

Example 4. Fig. 4 shows examples of variational interpolation of convex polyhedra in the Euclidean plane and in Euclidean three-space. The bold curve is the variational interpolant. The shape of each polyhedron is determined by a certain number of random half-spaces which touch a given sphere.

The algorithm which was used for computing the variational interpolant is a very intuitive and geometric one, and can be followed in Fig. 2. It starts with any interpolant $I\left(p_{0}, \ldots, p_{n}\right)$ with $p_{i} \in X_{i}$ and marches, towards lower values of $q$. Selected intermediate interpolants are shown as dotted curves. The minimizing interpolant is shown in bold. It is described in [9]. In this way minimizing the function $F$ is done by minimizing the quadratic function $q$ in the convex polytope $X_{0} \ldots X_{n}$ - the jump conditions of Th. 4 are not used in the computation.

\section{Closed Interpolants}

The topics discussed in this paper could be repeated for closed curves, which are easier to handle in the equivalent form of periodic curves

$$
c: \mathbb{R} \rightarrow \mathbb{R}^{d}, \quad c(t+T)=c(t) \text { for all } t \in \mathbb{R} .
$$

For the convenience of the reader, we explicitly state some definitions and results here. 
We start with periodic parameter values

$$
\ldots, u_{0}, u_{1}, \ldots, u_{n}=u_{0}+T, u_{n+1}=u_{1}+T, \ldots
$$

and consider the linear spaces $\mathcal{C}_{c}$ of $T$-periodic curves which otherwise have the same properties as the curves of $\mathcal{C}$. The definition of $F$ is literally the same as given by Equ. (4). The list of subsets to be interpolated is extended periodically by letting $X_{i+n}=X_{i}$ for all $i$. The definition of minimizing interpolant is obvious.

5.1. Affine subspaces. Lemma 1 concerning the variational interpolant of subspaces remains valid, if the special cases $i=0$ and $i=n$ in (c) are discarded and the orthogonality condition is required to hold for all $n$.

The definition of ' $\mathcal{P}_{c}^{3}$ ' is postponed until later. Lemma 2 assumes the following form:

Lemma 12. (Lemma 2, closed version) Assume that $X_{i}=p_{i}+U_{i}$ are affine subspaces of $\mathbb{R}^{d}$. If $U_{1} \cap \cdots \cap U_{n}=\{0\}$, then there is a unique closed minimizing interpolant of $X_{1}, \ldots, X_{n}$, and it satisfies (a)-(b) (appropriately modified) of Lemma 1.

Proof. The proof of Lemma 2 is modified in the following way: Instead of the end conditions we have $3 d$ 'closure conditions' $c\left(u_{0}\right)=c\left(u_{n}\right)$, $c^{\prime}\left(u_{0}\right)=c^{\prime}\left(u_{n}\right), c^{\prime \prime}\left(u_{0}\right)=c^{\prime \prime}\left(u_{n}\right)$. Again we have $\left(\operatorname{dim} \mathcal{P}^{3}\right)$ linear equations.

We get the corresponding homogeneous system if we replace $X_{i}$ by $U_{i}$ and look for closed minimizing interpolants of $U_{1}, \ldots, U_{n}$. Again these must be linear. The only closed curves among them are the constant curves, that is, the solution is unique if $U_{1} \cap \cdots \cap U_{n}=\{0\}$.

For points $p_{1}, \ldots, p_{n}$ there is a unique closed minimizing interpolant, which is $C^{2}$ and piecewise cubic. It is denoted by

$$
I_{c}\left(p_{1}, \ldots, p_{n}\right) .
$$

An example is shown in Fig. 5.

Lemma 3 remains true. We additionally are able to define a linear space $\mathcal{P}_{c}^{3}$ of closed $C^{2}$ piecewise cubic curves. Clearly,

$$
\operatorname{dim} \mathcal{P}_{c}^{3}=n d,
$$

which is an analogue to Equ. (10).

5.2. Submanifolds. As to submanifolds of Euclidean space, part (b) of Th. 4 has to be discarded, and the orthogonality condition of part (a) now reads ' $\Delta_{u_{i}} c^{\prime \prime \prime} \perp T_{c\left(u_{i}\right) X_{i}}$, for $i=1, \ldots, n$ '. In Th. 5 and Th. $6 \mathcal{C}$ and $\mathcal{P}^{3}$ have to replaced by $\mathcal{C}_{c}$ and $\mathcal{P}_{c}^{3}$, resp. 
5.3. Existence and uniqueness. The discussions concerning the existence and uniqueness of the minimizing interpolant in Sec. 4 are modified in a straightforward way. We define

$$
V^{c}=\left(\mathbb{R}^{d}\right)^{n}, \quad q_{c}\left(p_{1}, \ldots, p_{n}\right)=F\left(I_{c}\left(p_{1}, \ldots, p_{n}\right)\right) .
$$

The quadratic form $q_{c}$ is positive semidefinite. It defines a scalar product $\langle,\rangle_{c}$ in $V_{c}$, whose radical is given by

Lemma 13. (Lemma 7 , closed version)

$$
V_{c}^{\perp}=\left\{(p, \ldots, p) \mid p \in \mathbb{R}^{d}\right\}, \quad \operatorname{dim} V_{c}^{\perp}=d .
$$

This follows from the description of closed curves with $F(c)=0$ (which turn out to be constant; see the proof of Lemma 12).

Lemma 8, which expresses the solvability of the minimization problem, remains valid. Th. 9 assumes the following form:

Theorem 14. (Th. 9, closed version) Affine subspaces $X_{i}=p_{i}+U_{i}$ possess a closed minimizing interpolant. All minimizing interpolants are translates of each other, and they are of the form $I_{c}\left(x_{0}, \ldots, x_{n}\right)$. The set of translation vectors equals $U_{1} \cap \cdots \cap U_{n}$.

Proof. The proof is completely analogous to the proof of Th. 9. The only difference is the form of $V_{c}^{\perp}$ compared to $V^{\perp}$. As $V_{c}^{\perp}$ consists of the vectors $(p, \ldots, p)$, with $p \in U_{1} \cap \cdots \cap U_{n}$, the statement follows in a way completely analogous to the 'non-closed' version.

Cor. 10 (concerning the existence of a minimizing interpolant for polyhedra) need no change. The 'closed' form of Th. 11 is the following Theorem 15. (Th. 11, closed version) If $X_{0}, \ldots, X_{n}$ are convex polyhedra, the variational interpolation problem has a solution of the form $I_{c}\left(p_{0}, \ldots, p_{n}\right)$, with $p_{i} \in X_{i}$. All solutions are translates of each other, and have the form $I\left(x_{0}, \ldots, x_{n}\right)$, where $x_{i} \in X_{i}, x_{i}-p_{i}=p, p \in$ $U_{1} \cap \cdots \cap U_{n}$.

The proof is the same as for Th11, with the obvious modifications ( $V_{c}^{\perp}$ instead of $V^{\perp}$, as in the proof of Th. 14).

\section{Some Extensions and Related Topics}

6.1. Variational interpolation on surfaces. The problem of making the functional $\int\left\|c^{\prime \prime}\right\|^{2}$ stationary can be posed in a Riemannian manifold. It turns out that Th. 4 is valid with the difference that the curve segments are not piecewise cubic (which would mean $c^{\prime \prime \prime \prime}=0$ ), but satisfy the differential equation $c^{\prime \prime \prime \prime}+R\left(c^{\prime}, c^{\prime \prime}\right) c^{\prime}=0$. Here the prime denotes covariant differentiation and $R$ is the Riemannian curvature tensor. For a proof, see [16].

The original reason why the function $F$ was considered in the CAGD community was the fact that it is an easy-to-handle approximation of the functional $\int \kappa^{2} d s$, where $\kappa$ is the curvature of a curve, and $d s$ its arc length differential. For curves on a surface, the approximation is true 
to the same extent as in Euclidean space. Unfortunately the 'simplified' functional does not lead to an explicit solution or to a differential equation which is easy to handle.

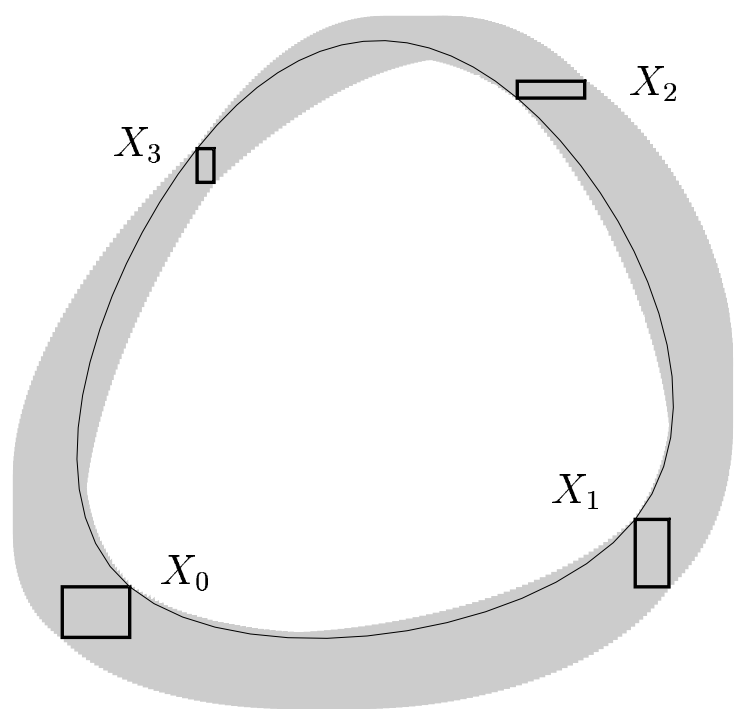

Figure 5 . The area traced out by $I_{c}\left(X_{0}, \ldots, X_{3}\right)$ for rectangles $X_{i}$.

6.2. Approximation curves with prescribed error bounds. The minimization problem

$$
\left\|p_{0}-c\left(u_{0}\right)\right\| \leq \rho_{0}, \ldots\left\|p_{n}-c\left(u_{n}\right)\right\| \leq \rho_{n}, \quad F(c) \rightarrow \text { min, }
$$

with prescribed nonnegative real numbers $\rho_{i}$ is the same as the problem of variational interpolation considered above, with $X_{i}=p_{i}+\rho_{i} B^{d}$, where $B^{d}$ stands for the closed unit ball of $\mathbb{R}^{d}$. This is the problem extensively discussed in [4]. A related construction which however does not give exact error bounds is the smoothing spline (cf. [14]).

6.3. Interval interpolation. The manipulation of imprecisely defined numbers, or more specifically, numbers which are contained in certain intervals, is the object of study in the field of interval arithmetic (a general reference is e.g., [8], more specific references for splines and their connection to interval arithmetic are $[1,2,13])$.

If the numbers in question are coordinates of points, these points are confined to rectangles, or boxes of dimension $d$. These in turn are convex polyhedra. In this way some questions of interval arithmetic are special cases of questions treated in this paper. We use the notation

$$
x_{i}=\left(x_{i}^{1}, \ldots, x_{i}^{d}\right)
$$

for coordinates of points and assume that

$$
x_{i}^{j} \in I_{i}^{j}, \quad I_{i}^{j}=\left[a_{i}^{j}, b_{i}^{j}\right],
$$


where $I_{i}^{j}$ is the interval which contains the $j$-th coordinate of the $i$-th point. Then we let

$$
X_{i}=I_{i}^{1} \times \cdots \times I_{i}^{d}
$$

Several questions may be asked: If points $p_{0}, \ldots, p_{n}$ are confined to boxes $X_{0}, \ldots, X_{n}$, what is the region traced out by all possible minimal interpolants $I\left(x_{0}, \ldots, x_{n}\right)$ for $x_{i} \in X_{i}$, or the same question for closed curves $I_{c}\left(x_{1}, \ldots, x_{n}\right)$ ? This question has been addressed in [15]. Fig. 5 shows an example.

Another question is which of the possible minimizing interpolants actually minimizes the functional $F$. This is nothing but variational interpolation of the boxes $X_{i}$. An example is shown by Fig. 2 .

A third question is which points $x_{i} \in X_{i}$ maximize $F\left(I\left(x_{0}, \ldots\right.\right.$, $\left.\left.x_{n}\right)\right)=q\left(x_{0}, \ldots, x_{n}\right)$. This amounts to finding the maximum of a quadratic function in a polyhedron (which in this case is the product of intervals). Fortunately the restriction of a positive semidefinite quadratic form to an affine subspace does not have a maximum unless it is constant. Thus the maximum in question is attained in a vertex of $X_{0} \times \cdots \times X_{n}$ :

$$
\max _{x_{i} \in X_{i}} q\left(x_{0}, \ldots, x_{n}\right)=\max _{x_{i}^{j}=a_{i}^{j}, b_{i}^{j}} q\left(x_{0}, \ldots, x_{n}\right) .
$$

It follows that the actual computation of this maximum amounts to at most $2^{(n+1) d}$ evaluations of $q$.

\section{ACKNOWLEDGEMENTS}

The authors are grateful to the anonymous referees for their careful reading of the manuscript and for pointing out references to previous work.

The algorithm used for minimizing a quadratic form on a convex polyhedron was implemented by N. Pomaroli some time ago, then serving for a completely different purpose, while he was supported by Project No. 12252-MAT of the Austrian Science Foundation.

The explicit system of equations for variationally interpolating lines in three-space has been implemented by B. Odehnal, whose work was supported by Project No. P-13648-MAT of the Austrian Science Foundation.

\section{REFERENCES}

[1] F. Chen, W. Lou: Degree reduction of interval Bézier curves. Computer-Aided Design 32 (2000), 571-582.

[2] Hu C.-Y., T. Maekawa, E. C. Sherbrooke, N. M. Patrikalakis. Approximation of measured data with interval B-splines. Computer-Aided Design 20 (1997), 791-799.

[3] Kersey, S. N.: Best near-interpolation by curves: existence. SIAM J. Numer. Anal. 38 (2000), 1666-1675. 
[4] Kersey, S. N.: Near-interpolation. To appear in Numerische Mathematik (2003).

[5] Kersey, S. N.: Best near-interpolation by curves: existence and convergence. preprint, University of Wisconsin, Madison. Available from http://www.cs.wisc. edu/ deboor/ftpreadme.html

[6] Kersey, S. N.: Near-interpolation to arbitrary constraints. To appear in the proceedings of the Fifth International Conference on Curves and Surfaces, Saint-Malo 2002 (2003).

[7] Kersey, S. N.: On the problems of smoothing and near-interpolation. To appear in Mathematics of Computation (2003).

[8] R. E. Moore, Interval Analysis, Prentice Hall, Englewood Cliffs, NJ, 1966.

[9] H. Pottmann, J. Wallner, Approximation Algorithms for Developable Surfaces. Comput. Aided Geometric Design 16 (1999), 539-556.

[10] H. Pottmann, J. Wallner, Computational Line Geometry, Springer, 2001.

[11] W. Rudin, Real and Complex Analysis. Third Ed., MacGraw-Hill, 1987.

[12] L. L. Schumaker: Spline Functions: Basic Theory, Wiley, New York, 1981.

[13] Sederberg T. W. and R. T. Farouki. Approximation by interval Bézier curves. IEEE Computer Graphics 12 (1992), 87-95.

[14] F. Utreras: Cross validation techniques for smoothing spline functions in one or two dimensions, in: M. Rosenblatt and Th. Gasser (eds.): Smoothing Techniques for Curve Estimation (Lecture Notes in Math. 757), Springer Verlag 1979, pp. 196-232.

[15] J. Wallner, R. Krasauskas, H. Pottmann, Error Propagation in Geometric Constructions, Computer-Aided Design 32 (2000), 631-641.

[16] J. Wallner, H. Pottmann: Variational Interpolation. Technical Report No. 84, Institut für Geometrie, Technische Universität Wien, June 2001. Available from http://www.geometrie.tuwien.ac.at/wallner/publ.html

\section{Authors' ADDRESS:}

Johannes Wallner, Helmut Pottmann

Institut für Geometrie

Technische Universität Wien

Wiedner Hauptstr. 8-10/113

A 1040 Wien

wallner@geometrie.tuwien.ac.at

pottmann@geometrie.tuwien.ac.at 